\title{
Systemic Inflammatory Biomarkers in Patients with Fuchs' Uveitis Syndrome: Neutrophil/Lymphocyte Ratio and Platelet/Lymphocyte Ratio
}

\author{
Sevcan Yildiz Balci, Alev Ozcelik Kose, Merve Beyza Yildiz and Sehnaz Ozcaliskan \\ Department of Ophthalmology, University of Health Sciences, Haydarpasa Numune Training and Research Hospital, Istanbul, Turkey
}

\begin{abstract}
Objective: To evaluate the neutrophil to lymphocyte ratio (NLR) and platelet to lymphocyte ratio (PLR) in patients with Fuchs' uveitis syndrome (FUS).

Study Design: A case-control study.

Place and Duration of Study: Department of Ophthalmology, Haydarpasa Numune Training and Research Hospital, Turkey, between July 2016 and June 2019.

Methodology: Twenty-eight patients with unilateral FUS and 30 healthy subjects were enrolled in the study. The NLR and PLR were calculated from complete blood counts tests; and compared between the groups.

Results: There were 28 patients (14 females, 14 males; median age: 33.5 (30-47.5) in the FUS group; and 30 patients (16 females, 14 males; median age 37.5 (33-41.8) in the healthy group. The NLR (1.7 v.s 1.2, $p=0.036$ was found to be significantly higher and PLR was lower (100.27 \pm 37.13 v.s. $141.68 \pm 26.50 p<0.001)$ in the FUS group compared with the healthy controls. The median WBC ( $<<0.001)$, neutrophil $(p<0.001)$, Iymphocytes $(p<0.001)$, and monocyte $(p=0.041)$ values were found significantly higher in the FUS group compared with controls. The significant correlations were not seen between the number of anterior chamber cells; which are ocular inflammatory parameter and NLRs $(r=0.312, p=0.106)$ and PLRs $(r=0.148, p=0.453)$.

Conclusion: The current study suggests that a slowly progressive immune-mediated process can affect peripheral blood inflammatory biomarkers and there is evidence of benign subclinical systemic inflammation in patients with FUS.
\end{abstract}

Key Words: Fuchs uveitis syndrome, Neutrophil to lymphocyte ratio, Platelet to lymphocyte ratio.

How to cite this article: Balci SY, Kose AO, Yildiz MB, Ozcaliskan S. Systemic Inflammatory Biomarkers in Patients with Fuchs' Uveitis Syndrome: Neutrophil/Lymphocyte Ratio and Platelet/Lymphocyte Ratio. J Coll Physicians Surg Pak 2020; 30(07):722-725.

\section{INTRODUCTION}

Fuchs' uveitis syndrome (FUS) is a low grade, chronic uveitis that is most commonly presented unilaterally and accounts for $1-8 \%$ of all uveitis causes. ${ }^{1-4}$ Patients usually remain asymptomatic for years. Although its etiology is still unknown, recently published studies have reported that there is a strong association between FUS and several infectious agents, especially rubella virus. ${ }^{5-9}$ Diagnostic criteria include stellate keratic precipitates diffusely scattered through the corneal endothelium, chronic mild anterior chamber reaction, iris stromal atrophy, heterochromia, a cluster of cells in the anterior vitreous condensation and presence of condensation, and lack of posterior synechia. The disease was diagnosed clinically and there was no specific laboratory test for the diagnosis.

Correspondence to: Sevcan Yildiz Balci, Department of Ophthalmology, University of Health Sciences, Haydarpasa Numune Training and Research Hospital, Istanbul, Turkey

E-mail: svcnyldz@hotmail.com

Received: April 11, 2020; Revised: June 30, 2020;

Accepted: July 06, 2020

DOI: https://doi.org/10.29271/jcpsp.2020.07.722
Recent studies have reported that neutrophil to lymphocyte ratio (NLR) and platelet to lymphocyte ratio (PLR) can be useful inflammatory biomarkers for ocular diseases like uveitis, keratoconus, retinal vein occlusion, and age-related macular degeneration. ${ }^{10-12}$ However, to the best of authors' knowledge, NLR and PLR have not previously studied in patients with unilateral FUS. Therefore, the aim of this study was to investigate NLRs and PLRs in patients with this chronic inflammatory ocular disease in order to assess a possible relationship with relatively novel systemic inflammatory biomarkers.

\section{METHODOLOGY}

This study was conducted in accordance with the amended Declaration of Helsinki; and ethical clearance was obtained from the Human Research Ethics Committee of the Haydarpasa Numune Training and Research Hospital. All mandatory laboratory health and safety procedures were complied with throughout the course of the laboratory work. The medical records of 32 patients with FUS, who were seen at the Department of Ophthalmology, Haydarpasa Numune Training and Research Hospital between July 2016 and June 2019 were reviewed. Four patients who had bilateral FUS and were diagnosed with multiple sclerosis were excluded from the study, and 28 patients with unilateral FUS and 30 age matched control participants were included in the study. 
Table I: Comparison of demographic features and laboratory parameters between the groups.

\begin{tabular}{|c|c|c|c|}
\hline & $\begin{array}{l}\text { Patients with FUS }(n=28) \\
\text { Mean } \pm \text { s.d. / } n-\% \text { median (IQR) }\end{array}$ & $\begin{array}{c}\text { Controls }(n=30) \\
\text { Mean } \pm \text { s.d. } / \mathrm{n}-\% \text { median (IQR) }\end{array}$ & $\mathbf{p}$ \\
\hline Age & $33.5(30-47.5)$ & $37.5(33-41.8)$ & $0.602^{\$}$ \\
\hline Gender: Female/Male & $14(50) / 14(50)$ & $16(53.3) / 14(46.7)$ & $0.800 !$ \\
\hline WBC $\left(10^{3} / \mathrm{uL}\right)$ & $8(7.8-9.8)$ & $5.5(5.4-5.6)$ & $<0.001^{\$}$ \\
\hline Neutrophil $\left(10^{3} / \mathrm{uL}\right)$ & $4.8(4.0-5.4)$ & $3.3(3.3-3.4)$ & $<0.001^{\$}$ \\
\hline Lymphocyte (103/uL) & $2.7(2.2-3.3)$ & $1.9(1.8-1.9)$ & $<0.001^{\$}$ \\
\hline Memocytes $\left(10^{3} / \mathrm{uL}\right)$ & $0.5(0.4-0.6)$ & $0.4(0.4-0.5)$ & $0.041^{\$}$ \\
\hline Platelet $\left(10^{3} / \mathrm{uL}\right)$ & $250(219.3-291.5)$ & $259(236-297.5)$ & $0.418^{\$}$ \\
\hline NLR & $1.7(1.3-2.4)$ & $1.2(1.2-1.8)$ & $0.036^{\$}$ \\
\hline PLR & $100.27 \pm 37.13$ & $141.68 \pm 26.50$ & $<0.001^{\$}$ \\
\hline
\end{tabular}

Table II: Correlation of anterior chamber cell grading with NLR/PLR values in FUS group.

\begin{tabular}{|l|c|c|c|}
\hline & & NLR & PLR \\
\hline Anterior chamber cell & $\mathrm{r}$ & 0.312 & 0.148 \\
\hline Grading & $\mathrm{p}$ & 0.106 & 0.453 \\
\hline
\end{tabular}

Spearman Correlation; NLR: Neutrophil to lymphocyte ratio; PLR: Platelet to lymphocyte ratio; FUS: Fuchs' uveitis syndrome.

Informed consent to participate in the study was obtained from each participant. The group of healthy controls was formed by subjects who applied for a job in the ophthalmology Department and underwent health control examinations.

The diagnosis of FUS was based on typical clinical findings (characteristic diffuse stellate keratic precipitates, iris atrophy, heterochromia, iris nodules, vitreous cells, the lack of posterior synechia) by slit-lamp biomicroscopy. Laboratory investigations and complete blood counts (CBC) were evaluated in the patients with FUS and controls, and the grading of cellular reaction in the anterior chamber was assessed using the SUN (Standardisation of Uveitis Nomenclature) Working Group criteria in the patients with FUS. $^{13}$

Complete blood count tests were taken between 9.00 a.m. and 2.00 p.m. after overnight fasting from the antecubital veins of all study participants. The samples were analysed within 6 hours after sampling with Cell-Dyn 3700 Hematology Analyzer (Cell-Dyn 3700, Abbott Diagnostics, Abbott Park, IL, USA).

Exclusion criteria were the presence of ocular and systemic diseases which can affect the laboratory parameters.

Levels of white blood cells (WBC), neutrophils, lymphocytes, monocytes, and platelets were determined from the CBC.
The NLR and PLR were evaluated as the ratios of neutrophils to lymphocytes and platelets to lymphocytes, respectively.

The SPSS 22.0 (SPSS Inc., Chicago, IL, USA) program was used for the analyses. The Shapiro-Wilk test was used to measure the distribution of variables. The parametric Student's t-test and the nonparametric Mann-Whitney U-test were used for comparison of independent groups. The Chisquare test was used to compare categorical variables between the groups. Spearman's rho correlations were performed in order to assess the relationship between parameters. Data were reported as mean \pm standard deviation, median (IQR) number and percentage. A p-value of $<0.05$ was considered to be significant.

\section{RESULTS}

There were 28 (48.3\%) patients (14 females, 14 males; median (IQR) age: 33.5 (30-47.5) in the FUS group and 30 (51.7\%) patients (16 females, 14 males; median (IQR) age: 37.5 (33-41.8) in the healthy group. There was no statistically significant difference between the groups in the average age $(p=0.602)$ and gender distribution $(p=0.800)$. The distribution of the demographic characteristics of the groups is given in Table I.

The median WBC ( $p<0.001)$, neutrophil $(p<0.001)$, lymphocytes $(p<0.001)$, and monocyte $(p=0.041)$ values were found to be significantly higher in the FUS group compared with controls. The statistically significant differences were also found in the NLR $(p=0.036)$ and PLR $(p<0.001$ ) between the groups (Table I).

Spearman's rho correlation analyses between the anterior chamber grading and the NLR and PLR in the FUS group are presented in Table II.

\section{DISCUSSION}


To the best of authors' knowledge, the current study is the first to determine the NLR and PLR in patients with unilateral FUS and healthy subjects. The results show that patients with unilateral FUS had increased NLR and lower PLR values when compared with the controls. Studies on inflammatory biomarkers in ocular and systemic diseases have been conducted in recent years. ${ }^{10-12}$ It is well known that leucocytosis, neutrophils, and lymphocytes are involved in the systemic and local inflammatory responses, and there is an association between bacterial infection and leucocytosis, and between viral infection and lymphocytosis. ${ }^{14}$

Recent studies have shown that samples from aqueous humour have antibodies mostly to rubella virus, herpes simplex virus, cytomegalovirus, or toxoplasma gondii, and that these pathogens seem to persist in eyes with FUS. ${ }^{8,15,16}$ In the last decade, studies have indicated that the chronic inflammation of eyes with FUS is related mostly to rubella virus persistence in the intraocular fluid rather than to immune-mediated cytokines. ${ }^{16}$ De Groot-Mijnes et al. reported that one patient with FUS and blood-aqueous barrier breakdown had high levels of serum anti-rubella IgG. ${ }^{9}$ Based on the chronic pattern of the disease, the present study aimed to determine whether FUS can affect the levels of systemic inflammation markers in the peripheral blood of patients with FUS. The results of this study showed that the mean WBC, neutrophil, lymphocyte, and monocyte values and the NLR were significantly higher in FUS patients ( $p<0.05$ ) compared with the healthy subjects. Our hypothesis is that damage of the blood-aqueous barrier due to chronic inflammation can promote increased levels of the parameters addressed in this study.

Recently, it has been reported that NLR and PLR can be used as parameters in uveitis and systemic inflammatory diseases. ${ }^{17,18}$ Ozgonul et al. found that the NLR and PLR were higher in patients with idiopathic acute anterior uveitis compared with control patients, and reported that these values could be used as a significant biomarker to determine disease prognosis. ${ }^{17}$ Avci et al. reported that the NLR and PLR were had higher levels in patients with Behçet's with anterior uveitis attack compared with patients without uveitis attack. ${ }^{19}$ In the present study, NLR and PLR were found significantly different between the groups. According to the results herein presented, the chronic inflammation of the eye in FUS patients seems to alter the benign character of these systemic peripheral blood parameters.

Some studies have reported that NLR levels may be an inflammatory biomarker also in ocular diseases, such as keratoconus, retinal vein occlusion, and age-related macular degener-ation. ${ }^{10-12}$ Kurtul et al. reported that patients with neovascular age macular degeneration had higher NLR and PLR than controls. ${ }^{20}$ This result is possibly related to angiogenesisrelated inflammatory mechanisms. Icel et al. investigated the relationship between high axial myopia and NLR and PLR and found that patients with high axial myopia had higher levels of NLR and PLR than emmetropic eyes. ${ }^{21}$ They reported chorioretinal changes that were seen in the degenerative myopic group, which might be as a result of inflammation. ${ }^{21}$ According to these reports, it seems that significant elevation of inflammatory markers in peripheral blood can be seen also in ocular diseases that are not associated with systemic involvement, which is consistent with our study results.

There are published studies also that show that cell-mediated and gene mechanisms play a role in the etiopathogenesis of FUS disease. ${ }^{22,23}$ Labalette et al. studied $\mathrm{T}$ cell repertoire diversity by screening the expression of $T$ cell receptor $B$ variable regions gene from the aqueous humour and peripheral blood cells in two patients with FUS. ${ }^{22}$ One patient showed the presence of a finite number of CD8 (+) CD28 (neg) T cell clonotypes in both aqueous humour and peripheral blood cells. They suggested an antigen-driven process and the involvement of $T$ cells might play a role in the pathogenesis of FUS. Spriewald et al. studied polymorphisms of the tumour necrosis factor (TNF) and cytotoxic T lymphocyte-associated protein 4 (CTLA4) genes, which were shown to play an important role in auto-immune as well as infectious diseases, and found that a certain genotype was more prevalent in patients with FUS compared with the controls. ${ }^{23}$ The authors speculate that the cellular immune response and clearing of rubella virus, which have been pointed out as the suspected triggers of FUS, may be impaired in these patients. The CTLA4 gene determines the resistance to infectious agents. Chronic inflammation of the eyes in patients with FUS was thought to affect systemic immune activity in individuals with genetic predisposition and to be the cause of the significantly higher levels of systemic inflammatory markers compared with those of the controls in the present study.

Systemic involvement is not expected in FUS. There are case reports of patients with FUS and Horner syndrome. ${ }^{24}$ The congenital rubella syndrome may present with delayed manifestations including endocrinopathies, fibromuscular dysplasia, and progressive rubella panencephalitis. ${ }^{25}$ Some authors suggested that FUS might be delayed manifestations of congenital rubella syndrome. ${ }^{25}$ The systemic effects of the significant changes in the NLR, which is a new inflammatory biomarker, herein observed in patients with FUS compared with the healthy subjects, should be investigated in further studies.

The limitations of this study are: the small sample size and the fact that the presence of rubella virus in the intraocular fluid and CTLA4 gene in peripheral blood has not been studied in FUS. Further prospective studies need to determine whether there is a relationship between the presence of rubella virus in the intraocular fluids and the inflammatory biomarkers in peripheral blood tested in the current study. Additionally, the change of parameters studied overtime should be supported by prospective studies in FUS.

\section{CONCLUSION}

The presented study showed that a slow progressive immunemediated process in the eyes affect the newly accepted peripheral blood inflammatory markers, and there is a subclinical systemic inflammation in patients with FUS. 


\section{ETHICAL APPROVAL:}

This study was conducted in accordance with the amended Declaration of Helsinki, and ethical clearance was obtained from the Human Research Ethics Committee of the Haydarpasa Numune Training and Research Hospital

\section{PATIENTS' CONSENT:}

Written informed consents were obtained from all the participants in the study.

\section{CONFLICT OF INTEREST:}

The authors declared no conflict of interest.

\section{AUTHORS' CONTRIBUTION:}

SB, AOK, MBY: Conducted data collection, drafting, analysis, and interpretation of data and manuscript writing.

SO: Helped in writing the manuscript.

SB, SO: Commented on an earlier version of the manuscript.

SB: Revised the manuscript critically for important intellectual content; approved the final version to be published.

\section{REFERENCES}

1. Kazokoglu H, Onal S, Tugal-Tutkun I, Mirza E, Akova Y, Özyazgan $Y$, et al. Demographic and clinical features of uveitis in tertiary centers in Turkey. Ophthalmic Epidemiol 2008; 15(5):285-93.

2. Rathinam SR, Namperumalsamy P. Global variation and pattern changes in epidemiology of uveitis. Indian J Ophthalmol 2007; 55(3):173-83.

3. Tugal-Tutkun I, Güney-Tefekli E, Kamaci-Duman F, Corum I. A cross-sectional and longitudinal study of fuchs uveitis syndrome in Turkish patients. Am J Ophthalmol 2009; 148(4):510-5.

4. Yalçındağ FN, Özdal PC, Özyazgan Y, Batıoğlu F, Tugal-Tutkun I. Demographic and clinical characteristics of uveitis in Turkey: The first national registry report. Ocul Immunol Inflamm 2018; 26(1):17-26.

5. Schwab IR. The epidemiologic association of Fuchs' heterochromic iridocyclitis and ocular toxoplasmosis. Am J Ophthalmol 1991; 111(3):356-62.

6. Arffa RC, Schlaegel TF. Chorioretinal scars in fuchs' heterochromic iridocyclitis. Arch Ophthalmol 1984; 102(8): 1153-5.

7. Barequet IS, Li Q, Wang Y, O'Brien TP, Hooks JJ, Stark WJ. Herpes simplex virus DNA identification from aqueous fluid in Fuchs heterochromic iridocyclitis. Am J Ophthalmol 2000; 129(5): 672-3.

8. Suzuki J, Goto H, Komase K, Abo H, Fujii K, Otsuki N, et al. Rubella virus as a possible etiological agent of Fuchs heterochromic iridocyclitis. Graefe's Arch Clin Exp Ophthalmol 2010; 248(10):1487-91.

9. De Groot-Mijnes JDF, De Visser L, Rothova A, Schuller M, Van Loon AM, Weersink AJL. Rubella virus is associated with Fuchs heterochromic iridocyclitis. Am J Ophthalmol 2006; 141(1):
212-4.

10. Karaca EE, Özmen MC, Ekici F, Yüksel E, Türkołlu Z. Neutrophilto-lymphocyte ratio may predict progression in patients with keratoconus. Cornea 2014; 33(11):1168-73.

11. Dursun A, Ozturk S, Yucel H, Ozec AV, Dursun FG, Toker MI, et al. Association of neutrophil/lymphocyte ratio and retinal vein occlusion. Eur J Ophthalmol 2015; 25(4):343-6.

12. Ilhan N, Daglioglu MC, Ilhan O, Coskun M, Tuzcu EA, Kahraman $\mathrm{H}$, et al. Assessment of neutrophil/lymphocyte ratio in patients with age-related macular degeneration. Ocul Immunol Inflamm 2015; 23(4):287-90.

13. Jabs DA, Nussenblatt RB, Rosenbaum JT, Group. S of UN (SUN) W. Standardisation of uveitis nomenclature for reporting clinical data. Am J Ophthalmol 2005; 140(3): 509-16.

14. Naess A, Nilssen SS, Mo R, Eide GE, Sjursen H. Role of neutrophil to lymphocyte and monocyte to lymphocyte ratios in the diagnosis of bacterial infection in patients with fever. Infection 2017; 45(3):299-307.

15. Stunf S, Petrovec M, Žigon N, Hawlina M, Kraut A, de GrootMijnes JDF, et al. High concordance of intraocular antibody synthesis against the rubella virus and fuchs heterochromic uveitis syndrome in Slovenia. Mol Vis 2012; 18:2909-14.

16. Kreps EO, Derveaux T, De Keyser F, Kestelyn P. Fuchs' Uveitis Syndrome: No Longer a Syndrome? Ocul Immunol Inflamm 2016; 24(3):348-57.

17. Ozgonul C, Sertoglu E, Ayyildiz O, Mumcuoglu T, Kucukevcilioglu M, Gokce G, et al. Novel biomarkers for patients with idiopathic acute anterior uveitis: Neutrophil to lymphocyte ratio and platelet to lymphocyte ratio. Int J Ophthalmol 2017; 10(2): 262-6.

18. Şahin M, Elbey B, Şahin A, Yüksel H, Türkcü FM, Çaça İ. Neutrophil-to-lymphocyte ratio and platelet-to-lymphocyte ratio in retinal vein occlusion. Clin Exp Opt 2019; 103(4): 490-4.

19. Avci A, Avci D, Erden F, Ragip E, Cetinkaya A, Ozyurt K, et al. Can we use the neutrophil-to-lymphocyte ratio, platelet-tolymphocyte ratio, and mean platelet volume values for the diagnosis of anterior uveitis in patients with Behcet's disease. Ther Clin Risk Manag 2017; 13:881-6.

20. Kurtul BE, Ozer PA. The relationship between neutrophil-tolymphocyte ratio and age-related macular degeneration. Korean J Ophthalmol 2016; 30(5):377-81.

21. Icel E, Ucak T, Karakurt Y, Yilmaz H, Tasli NG, Turk A. The relation of neutrophil to lymphocyte ratio and platelet to lymphocyte ratio with high axial myopia. Ocul Immunol Inflamm 2020; 28(3):396-401.

22. Labalette P, Caillau D, Grutzmacher C, Dessaint JP, Labalette M. Highly focused clonal composition of CD8+ CD28neg T cells in aqueous humor of Fuchs heterochromic cyclitis. Exp Eye Res 2002; 75(3):317-25.

23. Spriewald BM, Lefter C, Huber I, Lauer B, Wenkel H. A suggestive association of fuchs heterochromic cyclitis with cytotoxic T cell antigen 4 gene polymorphism. Ophthalmic Res 2007; 39(2):116-20.

24. Aggarwal RK, Luck J, Coster DJ. Horner's syndrome and Fuchs' heterochromic uveitis. BrJ Ophthalmol 1994; 78(12): 949.

25. O'Dea AF, Mayhall CA. Delayed manifestations of congenital rubella. J Vis Impair Blind 1988; 82(9):379-81. 